\title{
Erratum
}

\section{Erratum: Energy and angular distribution of electrons ejected from autoionization states in helium by electron impact [Phys. Rev. A 15, 574 (1977)]}

\section{N. Oda, S. Tahira, F. Nishimura, and F. Koike}

Values in the column labeled "Bhatia" are incorrect and two values should be added in the column labeled "Bhatia and Temkin" $\mathrm{h}$ " in Table II. Table II should be replaced by the following.

TABLE II. Resonance energies ( $E_{r}$ 's) of autoionization states in helium (in units of eV). The figures in brackets denote the expected error in the last digit of the experimental values.

\begin{tabular}{|c|c|c|c|c|c|c|c|c|c|c|c|}
\hline \multirow[b]{2}{*}{ State } & \multicolumn{5}{|c|}{ Experiment $\left(E_{r}\right)$} & \multicolumn{6}{|c|}{ Theory $\left(E_{r}\right)$} \\
\hline & $\begin{array}{l}\text { This } \\
\text { work }\end{array}$ & $\begin{array}{l}\text { Madden } \\
\text { and } \\
\text { Codling }{ }^{a}\end{array}$ & Rudd $^{\text {b }}$ & $\begin{array}{c}\text { Hicks } \\
\text { and } \\
\text { Comer }^{c}\end{array}$ & $\begin{array}{l}\text { Siegbahn } \\
\text { et al. }{ }^{\mathrm{d}}\end{array}$ & $\begin{array}{l}\text { Burke } \\
\text { and } \\
\text { Taylor } \mathrm{e}\end{array}$ & $\begin{array}{c}\text { Burke } \\
\text { and } \\
\text { McVicar }\end{array}$ & $\begin{array}{c}\text { Bhatia } \\
\text { and } \\
\text { Temkin }{ }^{\mathrm{g}}\end{array}$ & $\begin{array}{l}\text { Bhatia } \\
\text { and } \\
\text { Temkin }^{h}\end{array}$ & Bhatia $^{\text {i }}$ & $\begin{array}{l}\text { Altick } \\
\text { and } \\
\text { Moore } \mathrm{e}^{\mathrm{j}}\end{array}$ \\
\hline$\left(2 s^{2}\right)^{1} s$ & $57.84(2)$ & & $57.82(5)$ & $57.82(4)$ & $57.95(3)$ & 57.842 & 57.865 & & 57.844 & 57.836 & \\
\hline$(2 s 2 p)^{3} P$ & $58.30(2)$ & & $58.34(5)$ & $58.30(3)$ & & 58.317 & 58.360 & 58.298 & 58.321. & & \\
\hline$\left(2 p^{2}\right)^{1} D$ & $59.89(2)$ & & 60.0 & $59.89(3)$ & $59.86(2)$ & 59.911 & & & 59.915 & & \\
\hline$(2 s 2 p)^{1} P$ & 60.13 & $60.130(15)^{\mathrm{k}}$ & 60.13 & 60.130 & $60.12(1)$ & 60.149 & 60.269 & 60.143 & 60.145 & 60.146 & 60.340 \\
\hline$\left(2 p^{2}\right)^{1} s$ & $62.08(3)$ & & $62.15(5)$ & $62.06(3)$ & & 62.134 & 62.808 & & 62.091 & & \\
\hline$(2 s 3 s)^{1} S$ & $62.94(3)$ & & $62.95(5)$ & $62.94(3)$ & $62.94(2)$ & 62.975 & 63.009 & & 62.962 & & \\
\hline$(s p 23-)^{3} P^{\mathrm{m}}$ & $63.08(3)$ & & $63.08(5)$ & $63.07(3)$ & & & 63.141 & & 63.107 & & \\
\hline$(2 p 3 p)^{1} D$ & $63.52(3)$ & & & $63.50(3)$ & $63.50(2)$ & & & & 63.526 & & \\
\hline$(s p 23+)^{1} P$ & $63.64(3)$ & $63.651(7)^{1}$ & 63.65 & $63.65(3)$ & $63.65(2)$ & & 63.691 & 63.677 & 63.661 & & 63.707 \\
\hline$(2 p 3 p)^{1} S$ & $64.12(3)$ & & $64.22(5)$ & & & & 64.182 & & 64.101 & & \\
\hline$(2 s 4 s){ }^{1} S$ & $64.12(3)$ & & $64.22(5)$ & $64.18(3)$ & $64.22(3)$ & & 64.216 & & & & \\
\hline$(s p 24-)^{3} P^{\mathrm{m}}$ & $64.24(3)$ & & $64.22(5)$ & $64.23(3)$ & & & 64.255 & & & & \\
\hline$(2 p 4 p)^{1} D$ & $64.40(3)$ & & & $64.39(3)$ & $64.38(3)$ & & & & 64.415 & & \\
\hline$(s p 24+)^{1} P$ & $64.48(3)$ & $64.462(7)$ & $64.46(5)$ & $64.45(3)$ & $64.45(2)$ & & 64.481 & & & & \\
\hline$(2 s 5 s)^{1} S$ & $64.70(4)$ & & $64.71(5)$ & $64.67(4)$ & $64.70(2)$ & & 64.698 & & & & \\
\hline$(s p 25-)^{3} P^{\mathrm{m}}$ & $64.70(4)$ & & $64.71(5)$ & $64.69(4)$ & & & 64.712 & & & & \\
\hline$(2 p 5 p)^{1} D$ & $64.74(4)$ & & & & & & & & & & \\
\hline$(s p 25+)^{1} P$ & $64.80(3)$ & $64.813(7)$ & & & $64.81(3)$ & & $64: 824$ & & & & \\
\hline
\end{tabular}

${ }^{\mathrm{a}}$ Reference 1.

b Reference 7.

c. Reference 26 .

d Reference 23 .

e Reference 47.

f Reference 46 .

g A. K. Bhatia and A. Temkin, Phys. Rev. 182, 15 (1969).

h Reference 54.

i A. K. Bhatia, Phys. Rev. A 10, 729 (1974).

j P. L. Altick and E. N. Moore, Phys. Rev. Lett. 15, 100 (1965).

$\mathrm{k}$ This value was used to calibrate the energy scale of the present measurement.

1 This value was used to calibrate the energy scale by Rudd (Ref. 7).

${ }^{m}$ If we follow the notations by Altic and Moore ${ }^{j}$ and by L. Lipsky and A. Russek [Phys. Rev. 142, 59 (1966)], this series may be expressed as $(s p 2 n+)^{3} P(n=3,4,5)$.

If the energy calibration is done based on the best theoretical value by Temkin and Bhatia ${ }^{\mathrm{h}}(60.145 \mathrm{eV})$ for the $(2 s 2 p)^{1} P$ state in place of that by Madden and Codling $(60.130 \mathrm{eV})$ used in this work, all the experimental energies listed in the columns labeled "this work," "Rudd ${ }^{\mathrm{b}}$," and "Hicks and Comer " in Table II will be increased by $\sim 0.015 \mathrm{eV}$. The authors wish to thank Dr. A. K. Bhatia, Dr. A. Temkin, and Dr. W. Shearer-Izumi for their valuable comments. 